Groups Geom. Dyn. 7 (2013), 403-417

DOI $10.4171 / \mathrm{GGD} / 187$
Groups, Geometry, and Dynamics

(C) European Mathematical Society

\title{
Rigidity for equivalence relations on homogeneous spaces
}

\author{
Adrian Ioana and Yehuda Shalom*
}

\begin{abstract}
We study Popa's notion of rigidity for equivalence relations induced by actions on homogeneous spaces. For any lattices $\Gamma$ and $\Lambda$ in a semisimple Lie group $G$ with finite center and no compact factors we prove that the action $\Gamma \curvearrowright G / \Lambda$ is rigid. If in addition $G$ has property (T) then we derive that the von Neumann algebra $L^{\infty}(G / \Lambda) \rtimes \Gamma$ has property (T). We also show that if the stabilizer of any non-zero point in the Lie algebra of $G$ under the adjoint action of $G$ is amenable (e.g., if $G=\mathrm{SL}_{2}(\mathbb{R})$ ), then any ergodic subequivalence relation of the orbit equivalence relation of the action $\Gamma \curvearrowright G / \Lambda$ is either hyperfinite or rigid.
\end{abstract}

Mathematics Subject Classification (2010). 37A20, 46L36.

Keywords. Relative property $(\mathrm{T})$, homogenous spaces, $\mathrm{II}_{1}$ factors, equivalence relations.

\section{Introduction and statement of main results}

In [Po06], S. Popa introduced the notion of relative property $(T)$ for inclusions of finite separable von Neumann algebras $B \subset M-$ it has since been at the heart of his deformation/rigidity theory. When applied to inclusions arising from actions and equivalence relations, this concept suggested two new properties for actions and equivalence relations:

- A probability measure preserving (pmp) action $\Gamma \curvearrowright(X, \mu)$ of a countable group $\Gamma$ is rigid if the inclusion of $L^{\infty}(X)$ in the crossed-product algebra $L^{\infty}(X) \rtimes \Gamma$ ([MvN36]) has the relative property (T) in the sense of [Po06], Definition 4.2.1.

- A countable pmp equivalence relation $R$ on $(X, \mu)$ is rigid if the inclusion of $L^{\infty}(X)$ in the von Neumann algebra $L(R)$ of $R$ ([FM77]) has the relative property $(\mathrm{T})$.

Note that for free actions, rigidity is a property of their equivalence relations: a free pmp action $\Gamma \curvearrowright(X, \mu)$ is rigid if and only if its orbit equivalence relation $(x \sim y$ if $\Gamma x=\Gamma y)$ is.

* First author supported by a Clay Research Fellowship. Second author supported by NSF grants DMS 0701639 and 1007227. 
In the last decade these notions of rigidity have led to several remarkable applications, most notably, to calculations of invariants of von Neumann algebras and equivalence relations ([Po06], [PV10], [Ga11]) and to constructions of non-orbit equivalent actions of non-amenable groups ([GP05], [Io11]).

Yet, while rigidity was successfully exploited in applications, the theoretical aspects of its study (e.g., finding new constructions of rigid equivalence relations and a more manageable definition of rigidity - avoiding the use of von Neumann algebras) were neglected. In fact, until recently all known examples of rigid actions and equivalence relations ([Po06], [Ga11]) relied on the following group theoretic construction. Let $A$ be a countable abelian group together with an action of a countable group $\Gamma$. Then the pair $(\Gamma \ltimes A, A)$ has the relative property (T) of Kazhdan-Margulis if and only if the (Haar) measure preserving action $\Gamma \curvearrowright \widehat{A}$ and its orbit equivalence relation are rigid ([Po06]). In particular, since the pair $\left(\mathrm{SL}_{n}(\mathbb{Z}) \ltimes \mathbb{Z}^{n}, \mathbb{Z}^{n}\right)$ has the relative property $(\mathrm{T})$ ([Ka67], [Ma82]), it follows that the natural action of $\mathrm{SL}_{n}(\mathbb{Z})$ on the $n$-torus $\mathbb{T}^{n}$ is rigid for all $n \geqslant 2$.

The situation improved with the finding of an ergodic theoretic criterion for rigidity of pmp actions and equivalence relations ([Io10], see the next section). The criterion was then used to produce the first examples of rigid equivalence relations not built from a pair of groups with relative property (T): if $S$ denotes the orbit equivalence relation of the action $\mathrm{SL}_{2}(\mathbb{Z}) \curvearrowright \mathbb{T}^{2}$, then any ergodic non-hyperfinite subequivalence relation $R \subset S$ is rigid ([Io10], Theorem 0.1). Although this result provides new instances of rigidity, it has the disadvantage of being limited to a specific action.

In this paper, we work in the general framework of actions on homogeneous spaces and prove rigidity for the induced (sub)equivalence relations under fairly general assumptions. More precisely, we consider actions of countable subgroups $\Gamma<G$ on the homogeneous space $\left(G / \Lambda, m_{G / \Lambda}\right)$, where $G$ is a real algebraic group, $\Lambda<G$ is a lattice and $m_{G / \Lambda}$ is the unique $G$-invariant probability measure on $G / \Lambda$.

Our first result asserts that, under mild assumptions on $G$, the action of any lattice $\Gamma<G$ on $G / \Lambda$ is rigid.

Theorem A. Let $G$ be a real algebraic group with finite center, no proper normal co-compact algebraic subgroups, and no non-trivial algebraic homomorphism into $\mathbb{R}^{*}$.

If $\Gamma, \Lambda<G$ are lattices, then the pmp action $\Gamma \curvearrowright\left(G / \Lambda, m_{G / \Lambda}\right)$ is rigid.

Moreover, if $G$ has property $(T)$ (e.g., if $G$ is a connected semisimple Lie group with finite center whose simple factors have real-rank $\geqslant 2)$, then $L^{\infty}(G / \Lambda) \rtimes \Gamma$ has property $(T)$.

Property (T) for von Neumann algebras $M$ was introduced by Connes and Jones in [CJ85]. For a crossed-product algebra $L^{\infty}(X) \rtimes \Gamma$ coming from a pmp action, it is equivalent to having both that $\Gamma$ is a property ( $\mathrm{T}$ ) group and that the action $\Gamma \curvearrowright(X, \mu)$ is rigid. Because lattices inherit property $(\mathrm{T})([\mathrm{Ka} 67])$, this indicates how to deduce the last line of Theorem A. 
Theorem A implies that for any $n \geqslant 3$, the crossed product von Neumann algebra $L^{\infty}\left(\mathrm{SL}_{n}(\mathbb{R}) / \mathrm{SL}_{n}(\mathbb{Z})\right) \rtimes \mathrm{SL}_{n}(\mathbb{Z})$ has property $(\mathrm{T})$. This provides new examples of property $(\mathrm{T})$ von Neumann algebras that are not constructed from countable property (T) groups. For other such examples, coming from discrete quantum groups, see [Fi10].

As a consequence of Theorem A we also derive:

Corollary B. Let $G$ be as in Theorem $A$ and $\Gamma, \Lambda<G$ be lattices. Let I be a countable set on which $\Gamma$ acts with infinite orbits and $X_{0}$ be some probability space. Endow $X=X_{0}^{I}$ with the corresponding generalized Bernoulli $\Gamma$-action.

Then for any pmp $\Gamma$-space $Y$, any measurable quotient $\Gamma$-map $p: X \times Y \rightarrow G / \Lambda$ depends a.e. on the second coordinate only.

The main result of [Io10] shows that the orbit equivalence relation $S$ of the action $\mathrm{SL}_{2}(\mathbb{Z}) \curvearrowright \mathbb{T}^{2}$ satisfies the following "global" dichotomy: any ergodic subequivalence relation $R \subset S$ is either hyperfinite or rigid. Our second result establishes this dichotomy for many other actions, including the action of $\mathrm{SL}_{2}(\mathbb{Z})$ on $\mathrm{SL}_{2}(\mathbb{R}) / \mathrm{SL}_{2}(\mathbb{Z})$ :

Theorem C. Let $G$ be any of the groups $\mathrm{SL}_{2}(\mathbb{R}), \mathrm{SL}_{2}(\mathbb{C}), \mathrm{SL}_{2}(\mathbb{R}) \ltimes \mathbb{R}^{2}$ or $\mathrm{SL}_{2}(\mathbb{C}) \ltimes$ $\mathbb{C}^{2}$. Let $\Gamma<G$ be a countable discrete subgroup and $\Lambda<G$ be a lattice. Denote by $S$ the orbit equivalence relation of the action $\Gamma \curvearrowright\left(G / \Lambda, m_{G / \Lambda}\right)$.

Then any ergodic subequivalence relation $R \subset S$ is either hyperfinite or rigid.

Moreover, for any subequivalence relation $R \subset S$, we can find a measurable partition $G / \Lambda=X_{0} \cup X_{1}$ such that $X_{0}, X_{1}$ are $R$-invariant, $\left.R\right|_{X_{0}}$ is hyperfinite and $\left.R\right|_{X_{1}}$ is rigid.

The rest of the paper consists of two sections. In the next one, we recall two ergodic theoretic criteria for rigidity of actions and equivalence relations. In the last section, we use these criteria to prove Theorems A and C, and their more general versions, Theorems $\mathrm{D}$ and $\mathrm{E}$.

Acknowledgment. We would like to express our deep gratitude to Gregory Margulis who made a crucial contribution to the paper during his distinguished lecture series visit at UCLA.

\section{A criterion for rigidity}

Theorem 4.4 in [Io10] gives an ergodic theoretic formulation of rigidity for free ergodic actions. Also, Proposition 2.2 in [Io10] provides an ergodic theoretic criterion for rigidity of ergodic equivalence relations. In this section, we note that appropriate versions of these results - implicitly proved but not stated in [Io10] - hold without the freeness and the ergodicity assumptions. 
Throughout the paper, for a standard Borel space $X$, we denote by $B(X)$ the algebra of complex-valued bounded Borel functions on $X$ and by $\mathcal{M}(X)$ the space of Borel probability measures on $X$.

Proposition 1 (Equivalent formulation of rigidity for actions). A pmp action $\Gamma \curvearrowright$ $(X, \mu)$ of a countable group $\Gamma$ on a probability space $(X, \mu)$ is rigid if and only iffor any sequence of Borel probability measures $v_{n}$ on $X \times X$ satisfying

(1) $p_{*}^{i} v_{n}=\mu$ for all $n$ and $i=1,2$, where $p^{i}: X \times X \rightarrow X$ denotes the projection onto the $i$-th coordinate,

(2) $\int_{X \times X} f(x) g(y) d v_{n}(x, y) \rightarrow \int_{X} f(x) g(x) d \mu(x)$ for all $f, g \in B(X)$,

(3) $\left\|(\gamma \times \gamma)_{*} v_{n}-v_{n}\right\| \rightarrow 0$ for every $\gamma \in \Gamma$,

we have that $v_{n}(\Delta) \rightarrow 1$ (where $\Delta \subset X \times X$ denotes the diagonal).

Here, for a bounded signed Borel measure $v$ on $X \times X$, the norm $\|v\|$ is obtained by viewing $v$ as a linear functional on $B(X \times X)$.

Proposition 2 (Criterion for rigidity of equivalence relations). Let $R$ be a countable pmp equivalence relation on a probability space $(X, \mu)$. Assume that for any sequence of Borel probability measures $v_{n}$ on $X \times X$ satisfying (1), (2) and

$\left(3^{\prime}\right)\left\|(\theta \times \theta)_{*} v_{n}-v_{n}\right\| \rightarrow 0$ for every $\theta$ belonging to the group $[R]$ of automorphisms of $(X, \mu)$ whose graph is contained in $R$,

we have that $v_{n}(\Delta) \rightarrow 1$.

Then $R$ is rigid.

Before indicating how these propositions follow from [Io10], let us recall the notion of relative property $(\mathrm{T})$ for von Neumann algebras.

Definition ([Po06], Definition 4.2.1). Let $(M, \tau)$ be a von Neumann algebra with a normal faithful tracial state $\tau$ and $B \subset M$ a von Neumann subalgebra.

We say that $B \subset M$ has relative property $(T)$ if whenever $\mathscr{H}$ is a Hilbert $M$ bimodule and $\xi_{n} \in \mathscr{H}$ is a sequence of unit vectors satisfying

- $\left\langle x \xi_{n}, \xi_{n}\right\rangle=\left\langle\xi_{n} x, \xi_{n}\right\rangle=\tau(x)$ for all $x \in M$ and every $n \geqslant 1$ (tracial),

- $\left\|x \xi_{n}-\xi_{n} x\right\| \rightarrow 0$ for all $x \in M$ (almost central),

we can find $\eta_{n} \in \mathscr{H}$ such that $b \eta_{n}=\eta_{n} b$ for all $b \in B$ and every $n \geqslant 1$, and $\left\|\eta_{n}-\xi_{n}\right\| \rightarrow 0$.

Proof of Proposition 1. The proof of the "only if part" is identical to that of "(a) $\Longrightarrow$ (c)" in [Io10], Theorem 4.4 (which does not actually use the freeness and ergodicity assumptions).

The proof of the "if part" is implicitly contained in[Io10], Section 2, but for completeness, we give full details. Let $\Gamma \curvearrowright(X, \mu)$ be an action such that for any 
sequence of probability measures $v_{n}$ satisfying (1)-(3), we must have $v_{n}(\Delta) \rightarrow 1$. Denote $M=L^{\infty}(X) \rtimes \Gamma$ and let $\left\{u_{\gamma}\right\}_{\gamma \in \Gamma}$ be the canonical unitaries. To prove that the action is rigid, let $\mathscr{H}$ be a Hilbert $M$-bimodule and $\xi_{n} \in \mathscr{H}$ a sequence of tracial, almost central vectors.

Since $(X, \mu)$ is a standard probability space, we may assume that $X$ is a compact metric space. We denote by $C(X)$ the algebra of complex-valued continuous functions on $X$. The left-right actions of $C(X)$ on $\mathscr{H}$ induce a $\mathrm{C}^{*}$-algebra representation of $C(X \times X) \simeq C(X) \bar{\otimes}_{\max } C(X)$ into $\mathbb{B}(\mathscr{H})$. Let $E: \Omega \rightarrow \mathcal{P}(\mathcal{H})$ be the spectral measure giving this representation, where $\Omega$ is the Borel $\sigma$-algebra of $X \times X$ and $\mathcal{P}(\mathscr{H})$ denotes the set of projections in $\mathbb{B}(\mathscr{H})$ (see e.g. [Co99], Theorem 9.8). Thus, if $\pi: B(X \times X) \rightarrow \mathbb{B}(\mathscr{H})$ is defined by $\pi(f)=\int_{X \times X} f d E$ for all $f \in B(X \times X)$, then

$$
\pi\left(f_{1} \otimes f_{2}\right)(\xi)=f_{1} \xi f_{2} \quad \text { for all } f_{1}, f_{2} \in C(X) \text { and all } \xi \in \mathscr{H} \text {. }
$$

Next, let $v_{n} \in \mathcal{M}(X \times X)$ be given by the formula $\int_{X \times X} f d v_{n}=\left\langle\pi(f) \xi_{n}, \xi_{n}\right\rangle$ for all $f \in B(X \times X)$. Since $\xi_{n}$ is tracial, equation (a) implies that $v_{n}$ satisfies (1) for all $n \geqslant 1$.

Now, by approximating Borel functions with continuous functions (using e.g. Lemma 9.7 of [Co99]), we have that (a) holds for every $f_{1}, f_{2} \in B(X)$. Thus,

$$
\begin{aligned}
& \left|\int_{X \times X} f_{1}(x) f_{2}(y) d v_{n}(x, y)-\int_{X} f_{1}(x) f_{2}(x) d \mu(x)\right| \\
& \quad=\left|\left\langle f_{1} \xi_{n} f_{2}, \xi_{n}\right\rangle-\left\langle f_{1} f_{2} \xi_{n}, \xi_{n}\right\rangle\right| \leqslant\left\|f_{1}\right\|_{\infty}\left\|f_{2} \xi_{n}-\xi_{n} f_{2}\right\|,
\end{aligned}
$$

for all $f_{1}, f_{2} \in B(X)$. Since $\xi_{n}$ are almost central it follows that $v_{n}$ satisfy (2).

Finally, let us show that $v_{n}$ satisfy (3). Fix $\gamma \in \Gamma$ and recall that $u_{\gamma} f u_{\gamma}^{*}=f \circ \gamma^{-1}$ for all $f \in L^{\infty}(X, \mu)$. Then (a) gives that for every $f_{1}, f_{2} \in C(X)$ we have that

$$
\begin{aligned}
\int_{X \times X}\left[\left(f_{1} \otimes f_{2}\right) \circ(\gamma \times \gamma)^{-1}\right] d v_{n} & =\int_{X \times X}\left[\left(f_{1} \circ \gamma^{-1}\right) \otimes\left(f_{2} \otimes \gamma^{-1}\right)\right] d v_{n} \\
& =\left\langle\left(f_{1} \circ \gamma^{-1}\right) \xi_{n}\left(f_{2} \circ \gamma^{-1}\right), \xi_{n}\right\rangle \\
& =\left\langle u_{\gamma} f_{1} u_{\gamma}^{*} \xi_{n} u_{\gamma} f_{2} u_{\gamma}^{*}, \xi_{n}\right\rangle \\
& =\left\langle f_{1}\left(u_{\gamma}^{*} \xi_{n} u_{\gamma}\right) f_{2}, u_{\gamma}^{*} \xi_{n} u_{\gamma}\right\rangle \\
& =\left\langle\pi\left(f_{1} \otimes f_{2}\right)\left(u_{\gamma}^{*} \xi_{n} u_{\gamma}\right), u_{\gamma}^{*} \xi_{n} u_{\gamma}\right\rangle .
\end{aligned}
$$

By approximating Borel functions with continuous functions we derive that

$$
\int_{X \times X} f \circ(\gamma \times \gamma)^{-1} d v_{n}=\left\langle\pi(f)\left(u_{\gamma}^{*} \xi_{n} u_{\gamma}\right), u_{\gamma}^{*} \xi_{n} u_{\gamma}\right\rangle \text { for all } f \in B(X \times X) .
$$

Since $\|\pi(f)\| \leqslant\|f\|_{\infty}$ for all $f \in B(X \times X)$, and $\left\|u_{\gamma}^{*} \xi_{n} u_{\gamma}-\xi_{n}\right\| \rightarrow 0$, equation (b) implies that $v_{n}$ also satisfy condition (3).

Thus, we must have $v_{n}(\Delta) \rightarrow 1$. Define $\eta_{n}:=\pi\left(1_{\Delta}\right)\left(\xi_{n}\right) \in \mathscr{H}$. Since $f \eta_{n}=$ $\eta_{n} f$ for all $f \in L^{\infty}(X)$, and $\left\|\eta_{n}-\xi_{n}\right\|_{2}=\sqrt{1-v_{n}(\Delta)} \rightarrow 0$, we are done. 
Proof of Proposition 2. This is the same as the proof of the "if part" of Proposition 1, where we replace $M$ by $L(R)$ and the unitaries $\left\{u_{\gamma}\right\}_{\gamma \in \Gamma}$ by the unitaries $\left\{u_{\theta}\right\}_{\theta \in[R]}$.

\section{Proofs}

We begin by stating more general versions of Theorems A and C.

Theorem D. Let $G$ be a real algebraic group and $\Lambda \subset G$ be a lattice. Let $\Gamma \subset G$ be a countable subgroup and denote by $H$ its Zariski closure. Assume that $H$ has no proper normal co-compact algebraic subgroup and no non-trivial homomorphism into $\mathbb{R}^{*}$. Let $\eta$ be a $\Gamma$-invariant probability measure on $G / \Lambda$.

If the centralizer of $\Gamma$ (equivalently, of $H$ ) in $G$ is finite, then the pmp action $\Gamma \curvearrowright(G / \Lambda, \eta)$ is rigid.

In the case $\eta=m_{G / \Lambda}$, the converse is true: if the action $\Gamma \curvearrowright(G / \Lambda, \eta)$ is rigid, then the centralizer of $\Gamma$ in $G$ is finite.

Remark. Theorem $\mathrm{D}$ implies that for $\Gamma=\mathbb{F}_{2} \times \mathbb{Z}$ actions of the form $\Gamma \curvearrowright$ $\left(G / \Lambda, m_{G / \Lambda}\right)$ are never rigid. It would be interesting to decide whether $\Gamma$ admits a free ergodic rigid action at all. Note in this respect that the general question of characterizing non-amenable groups which admit free ergodic rigid actions ([Po06], Problem 5.10.2) remains open (see [Ga11] for a partial result).

Theorem E. Let $G$ be a real algebraic group, fix a Haar measure $m=m_{G}$ of $G$ and on $(G, m)$ consider the left-right multiplication action of $G \times G:\left(g_{1}, g_{2}\right) \cdot g=$ $g_{1} g g_{2}^{-1}$.

Let $\Gamma, \Lambda<G$ be two countable discrete subgroups and denote by $H, K$ their Zariski closures. Assume that the stabilizer of any non-zero point in the Lie algebra of $G$ under the adjoint actions of $H$ and $K$ is amenable.

Denote by $S$ the orbit equivalence relation of the action $\Gamma \times \Lambda \curvearrowright(G, m)$, i.e., $S=\{(x, y) \in G \times G \mid x \in \Gamma y \Lambda\}$. Let $X \subset G$ be a Borel set with $0<m(X)<\infty$.

Then, for any subequivalence relation $\left.R \subset S\right|_{X}=S \cap(X \times X)$, we can find an $R$-invariant measurable partition $X=X_{0} \cup X_{1}$ such that $\left.R\right|_{X_{0}}$ is hyperfinite and $\left.R\right|_{X_{1}}$ is rigid.

Remark. The assumption that $\Gamma, \Lambda<G$ are discrete is essential. To see this, let $G$ be a simple connected compact Lie group together with two countable subgroups $\Gamma, \Lambda$. Suppose that $\Gamma<G$ is dense and non-amenable. These assumptions imply that the action $\Gamma \times \Lambda \curvearrowright(G, m)$ is free ergodic and its equivalence relation $S$ is ergodic and non-hyperfinite.

We claim that $S$ is not rigid. Let $d$ be a $G \times G$-invariant metric on $G$ defining the topology. For $n \geqslant 1$, let $A_{n}=\left\{(x, y) \in G \times G \mid d(x, y)<\frac{1}{n}\right\}$ and set 
$v_{n}=\frac{\left.(m \times m)\right|_{A_{n}}}{(m \times m)\left(A_{n}\right)} \in \mathcal{M}(G \times G)$. Then $v_{n}$ is invariant under the diagonal product action of $G \times G$ on $G \times G:\left(g_{1}, g_{2}\right) \cdot(h, k)=\left(g_{1} h g_{2}^{-1}, g_{1} k g_{2}^{-1}\right)$ and its projection onto both coordinates is equal to $m$. Moreover, we have $\int_{G \times G} f_{1}(h) f_{2}(k) d v_{n}(h, k) \rightarrow$ $\int_{G} f_{1}(h) f_{2}(h) d m(h)$ for all $f_{1}, f_{2} \in B(G)$ (this is clear when $f_{1}$ is a continuous function; in general, use Lusin's theorem to approximate $f_{1}$ and the fact that the projection of $v_{n}$ onto the first coordinate is equal to $\left.m\right)$. Since $v_{n}(\{(h, h) \mid h \in$ $G\})=0$ for all $n$, Proposition 1 shows that the free action $\Gamma \times \Lambda \curvearrowright(G, m)$ is not rigid. By [Po06] it follows that $S$ is not rigid, which shows that the conclusion of Theorem $\mathrm{E}$ fails in this case.

Next, we introduce some notation that we will use in the proofs of Theorems D and E. Fix an unimodular real algebraic group $G$ and a Haar measure $m=m_{G}$.

- Denote by $g$ the Lie algebra of $G$ and by $\mathbf{P}(\mathfrak{g})=(\mathfrak{g} \backslash\{0\}) / \mathbb{R}^{*}$ the associated projective variety together with the map $p: \mathfrak{g} \backslash\{0\} \rightarrow \mathbf{P}(\mathfrak{g})$.

We endow $\mathfrak{g}$ and $\mathbf{P}(\mathfrak{g})$ with the adjoint $G$-action.

- Let $q: G \rightarrow g$ be a Borel map which is equal to the logarithm in some neighborhood $U$ of $1 \in G$.

- Next, define $r: G \times G \rightarrow G$ by $r(x, y)=x y^{-1}$.

- We can now set $\rho=p \circ q \circ r:(G \times G) \backslash \Delta \rightarrow \mathbf{P}(\mathfrak{g})$, where $\Delta=\{(x, x) \mid x \in G\}$.

- Finally, we let $\pi:(G \times G) \backslash \Delta \rightarrow G \times \mathbf{P}(\mathfrak{g})$ be given by $\pi(x, y)=(x, \rho(x, y))$.

Given a Borel subset $X \subset G$ we denote $\Delta_{X}=\Delta \cap(X \times X)$ and the projection onto the $i$-th coordinate by $p^{i}: X \times X \rightarrow X$.

Lemma F. Let $X \subset G$ be a Borel subset endowed with a Borel probability measure $\eta$. Let $c>0$. Suppose that $\mu_{n} \in \mathcal{M}(X \times X)$ is a sequence satisfying $p_{*}^{1} \mu_{n} \leqslant c \eta$, $\mu_{n}\left(\Delta_{X}\right)=0$ for all $n \geqslant 1$, and $\mu_{n}(A \times(X \backslash A)) \rightarrow 0$ for any Borel set $A \subset X$.

Let $\Sigma \subset G$ be a countable subgroup and $\phi_{1}, \phi_{2}: X \rightarrow \Sigma$ be two Borel maps. Denote by $D$ the set of $(x, y) \in(X \times X) \backslash \Delta_{X}$ such that $\rho\left(\phi_{1}(x) x \phi_{2}(x), \phi_{1}(y) y \phi_{2}(y)\right)=$ $\operatorname{Ad}\left(\phi_{1}(x)\right)(\rho(x, y))$.

Then $\lim _{n \rightarrow \infty} \mu_{n}(D)=1$.

Proof. We first claim that $\mu_{n}\left(\bigcup_{i=1}^{\infty}\left(B_{i} \times B_{i}\right)\right) \rightarrow 1$ for any Borel partition $\left\{B_{i}\right\}_{i=1}^{\infty}$ of $X$. For $k \geqslant 1$, set $X_{k}=\bigcup_{i=1}^{k} B_{i}$. Note that $(X \times X) \backslash \bigcup_{i=1}^{\infty}\left(B_{i} \times B_{i}\right) \subset$ $\bigcup_{i=1}^{k}\left(B_{i} \times\left(X \backslash B_{i}\right)\right) \cup\left(\left(X \backslash X_{k}\right) \times X\right)$. Since $\mu_{n}\left(B_{i} \times\left(X \backslash B_{i}\right)\right) \rightarrow 0$ for all $i$ and $p_{*}^{1} \mu_{n} \leqslant c \eta$, we deduce that

$$
\limsup \mu_{n}\left((X \times X) \backslash \bigcup_{i=1}^{\infty}\left(B_{i} \times B_{i}\right)\right) \leqslant c \eta\left(X \backslash X_{k}\right) \text { for all } k \geqslant 1 .
$$

Since $\left\{B_{i}\right\}_{i=1}^{\infty}$ is a partition of $X$, we obtain that $\eta\left(X_{k}\right) \rightarrow 1$, which proves our claim. 
Towards proving that $\mu_{n}(D) \rightarrow 1$, let $\varepsilon>0$. We can find a finite subset $F$ of $\Sigma$ such that $\eta\left(\left\{x \in X \mid \phi_{1}(x) \in F\right\}\right) \geqslant 1-\frac{\varepsilon}{c}$. Since $p_{*}^{1} \mu_{n} \leqslant c \eta$ it follows that $A=\left\{(x, y) \in X \times X \mid \phi_{1}(x) \in F\right\}$ satisfies $\mu_{n}(A) \geqslant 1-\varepsilon$, for all $n \geqslant 1$.

Next, since $q(x)=\log (x)$ for $x$ in a neighborhood of $1 \in G$, we obtain that for all $x$ in a, possibly smaller, neighborhood $V$ of 1 we have that $q\left(\gamma x \gamma^{-1}\right)=$ $\operatorname{Ad}(\gamma)(q(x))$ for every $\gamma \in F$. Let $B=\left\{(x, y) \in X \times X \mid x y^{-1} \in V\right\}$. Let $W$ be a neighborhood of $1 \in G$ such that $W W^{-1} \subset V$ and $h_{1}, h_{2}, . . \in G$ be a sequence such that $G=\bigcup_{i=1}^{\infty} W h_{i}$. For $i \geqslant 1$, define $B_{i}=\left(W h_{i} \backslash\left(\bigcup_{j=1}^{i-1} W h_{j}\right)\right) \cap X$. Since $\left\{B_{i}\right\}_{i=1}^{\infty}$ is a partition of $X$ and $\bigcup_{i=1}^{\infty}\left(B_{i} \times B_{i}\right) \subset B$, the above claim yields that $\mu_{n}(B) \rightarrow 1$.

Finally, let us show that $C=\left\{(x, y) \in X \times X \mid \phi_{1}(x)=\phi_{1}(y), \phi_{2}(x)=\phi_{2}(y)\right\}$ satisfies $\mu_{n}(C) \rightarrow 1$. This also follows from the above claim, after noticing that the sets $C_{\gamma_{1}, \gamma_{2}}=\left\{x \in X \mid \phi_{1}(x)=\gamma_{1}, \phi_{2}(x)=\gamma_{2}\right\}$, with $\gamma_{1}, \gamma_{2} \in \Sigma$, form a partition of $X$ and satisfy $C=\bigcup_{\gamma_{1}, \gamma_{2} \in \Gamma}\left(C_{\gamma_{1}, \gamma_{2}} \times C_{\gamma_{1}, \gamma_{2}}\right)$. Finally, it is easy to see that $A \cap B \cap C \subset D \cup \Delta_{X}$. Since lim inf $\mu_{n}(A \cap B \cap C) \geqslant 1-\varepsilon$ and $\varepsilon>0$ is arbitrary, the proof is complete.

Proof of Theorem D. Suppose first that the centralizer of $H$ in $G$ is finite. Let $X \subset G$ be a fundamental domain for the right $\Lambda$-action. Identify $G / \Lambda$ with $X$ via the map $G / \Lambda \ni x \Lambda \rightarrow x \Lambda \cap X \in X$. Under this identification, the corresponding $\Gamma$-action on $X$ is given by $\gamma \cdot x=\gamma x w(\gamma, x)$ for all $x \in X$ and $\gamma \in \Gamma$, where $\lambda=w(\gamma, x)$ is the unique element of $\Lambda$ such that $\gamma x \lambda \in X$.

Let $v_{n}$ be a sequence of Borel probability measures on $X \times X$ satisfying

(1) $p_{*}^{i} v_{n}=\eta$ for all $n$ and $i=1,2$.

(2) $\int_{X \times X} f(x) g(y) d v_{n}(x, y) \rightarrow \int_{X} f g d \eta$ for all $f, g \in B(X)$.

(3) $\left\|(\gamma \times \gamma)_{*} v_{n}-v_{n}\right\| \rightarrow 0$ for all $\gamma \in \Gamma$.

By Proposition 1 , to conclude that the action $\Gamma \curvearrowright(X, \eta)$ is rigid, it suffices to argue that $v_{n}\left(\Delta_{X}\right) \rightarrow 1$.

If this is false, then after passing to a subsequence we may assume that $c_{n}=1-$ $v_{n}\left(\Delta_{X}\right)$ verify $c=\inf c_{n}>0$. Define $\mu_{n} \in \mathcal{M}(X \times X)$ by $\mu_{n}(A)=c_{n}^{-1} v_{n}\left(A \backslash \Delta_{X}\right)$ for any Borel set $A \subset X \times X$. Then conditions (1) and (2) imply that $p_{*}^{1} \mu_{n} \leqslant c^{-1} \eta$ for all $n$, and $\mu_{n}(A \times(X \backslash A)) \rightarrow 0$ for any Borel set $A \subset X$. By applying Lemma $\mathrm{F}$ (to the subgroup $\Sigma<G$ generated by $\Gamma$ and $\Lambda)$, we obtain $\mu_{n}\left(\left\{(x, y) \in(X \times X) \backslash \Delta_{X} \mid\right.\right.$ $\rho(\gamma \cdot x, \gamma \cdot y)=\operatorname{Ad}(\gamma)(\rho(x, y))\}) \rightarrow 1$.

Also, condition (3) gives that $\left\|(\gamma \times \gamma)_{*} \mu_{n}-\mu_{n}\right\| \rightarrow 0$ for all $\gamma \in \Gamma$. Combining the last two facts yields that the probability measures $\zeta_{n}=\rho_{*} \mu_{n}$ on $\mathbf{P}(\mathrm{g})$ satisfy $\left\|\operatorname{Ad}(\gamma)_{*} \zeta_{n}-\zeta_{n}\right\| \rightarrow 0$ for all $\gamma \in \Gamma$. Since $\mathbf{P}(\mathfrak{g})$ is a compact metric space, the space $\mathcal{M}(\mathbf{P}(\mathfrak{g}))$, endowed with the weak-* topology induced by the embedding $\mathcal{M}(\mathbf{P}(\mathfrak{g})) \subset C(\mathbf{P}(\mathfrak{g}))^{*}$, is compact metrizable. Let $\zeta \in \mathcal{M}(\mathbf{P}(\mathfrak{g}))$ be a weak-* limit point of $\left\{\zeta_{n}\right\}_{n \geqslant 1}$. Since $\Gamma$ acts on $\mathbf{P}(\mathfrak{g})$ by homeomorphisms, we deduce that $\zeta$ is invariant under the adjoint action of $\Gamma$. 
Since the connected component of $H$ in the Zariski topology is a normal cocompact algebraic subgroup, the hypothesis implies that $H$ is connected as an algebraic group.

By applying [Sh99], Theorem 3.11 (which uses the fact that $H$ is connected as an algebraic group) we get that $\zeta$ is invariant under the adjoint action of $H$ and that $H$ has a normal co-compact subgroup which fixes every point in the support of $\zeta$. Now, the hypothesis forces that $H$ fixes every point in the support of $\zeta$. In particular, there exists $Y \in \mathfrak{g} \backslash\{0\}$ and a homomorphism $\chi: H \rightarrow \mathbb{R}^{*}$ such that $\operatorname{Ad}(\gamma)(Y)=\chi(\gamma) Y$ for all $\gamma \in H$. Since every such $\chi$ is trivial, we deduce that $Y$ is invariant under the adjoint action of $H$. Hence, for all $n$, we have that $y_{n}=\exp \left(\frac{Y}{n}\right) \in G$ commutes with $H$. Since $Y \neq 0$, this contradicts the assumption that the centralizer of $H$ in $G$ is finite.

For the converse, suppose that $\eta=m_{G / \Lambda}$ and that the action $\Gamma \curvearrowright(G / \Lambda, \eta)$ is rigid. By way of contradiction, if the centralizer of $H$ in $G$ is infinite, we can find a sequence of elements $x_{n} \in G \backslash\{1\}$ which commute with $H$ and converge to 1 . For every $n$, let $\nu_{n}$ be the pushforward of $\eta$ through the map $G / \Lambda \ni g \Lambda \rightarrow$ $\left(g \Lambda, x_{n} g \Lambda\right) \in G / \Lambda \times G / \Lambda$. Since $x_{n}$ and $\Gamma$ commute, $v_{n}$ is invariant under the diagonal $\Gamma$-action. It is also clear that the projection of $v_{n}$ onto both coordinates is equal to $\eta$.

Now, let $f_{1}, f_{2}$ be bounded Borel functions on $G / \Lambda$. Since $x_{n} \rightarrow 1$, it is easy to see that $\int_{G / \Lambda}\left|f_{2}\left(x_{n} x\right)-f_{2}(x)\right| d \eta(x) \rightarrow 0$. This implies that

$$
\begin{aligned}
\int_{G / \Lambda \times G / \Lambda} & f_{1}(x) f_{2}(y) d v_{n}(x, y)=\int_{G / \Lambda} f_{1}(x) f_{2}\left(x_{n} x\right) d \eta(x) \\
& \rightarrow \int_{G / \Lambda} f_{1}(x) f_{2}(x) d \eta(x) .
\end{aligned}
$$

Since the action $\Gamma \curvearrowright(G / \Lambda, \eta)$ is rigid, we conclude that $v_{n}(\Delta) \rightarrow 1$. Equivalently, $m\left(\left\{\left(g \in X \mid g^{-1} x_{n} g \notin \Lambda\right\}\right) \rightarrow 0\right.$ for every Borel set $X \subset G$ with $m(X)<$ $\infty$. On the other hand, as $x_{n} \rightarrow 1$, we have $m\left(\left\{\left(g \in X \mid g^{-1} x_{n} g \notin U\right\}\right) \rightarrow 0\right.$ for any neighborhood $U$ of 1 in $G$. Since $\Lambda$ is discrete, we deduce that $x_{n}=1$ for $n$ large enough, a contradiction.

Proof of Theorem E. We claim that it is sufficient to show that whenever $X \subset G$ is a Borel set with $m(X) \in(0, \infty)$ and $\left.R \subset S\right|_{X}$ is a non-rigid subequivalence relation, there exists an $R$-invariant Borel subset $X_{0} \subset X$ such that $m\left(X_{0}\right)>0$ and $\left.R\right|_{X_{0}}$ is hyperfinite.

Assuming this is true, let $\left.R \subset S\right|_{X}$ be a subequivalence relation. Let $X_{0} \subset X$ be a Borel subset of maximal measure such that $\left.R\right|_{X_{0}}$ is hyperfinite. Then the conclusion of Theorem $\mathrm{E}$ is equivalent to the equivalence relation $R_{0}:=\left.R\right|_{X \backslash X_{0}}$ being rigid. If $R_{0}$ is not rigid, then since $\left.R_{0} \subset S\right|_{X \backslash X_{0}}$, we could find $X_{1} \subset X \backslash X_{0}$ Borel with $m\left(X_{1}\right)>0$ such that $\left.R\right|_{X_{1}}=\left.R_{0}\right|_{X_{1}}$ is hyperfinite. This however would contradict the maximality of $X_{0}$. 
So, let $\left.R \subset S\right|_{X}$ be a non-rigid equivalence relation. Let $\eta=\left.m(X)^{-1} m\right|_{X}$ be the probability measure on $X$ obtained by normalizing the restriction of $m$ to $X$. Since $R$ is not rigid, Proposition 2 gives a sequence $v_{n} \in \mathcal{M}(X \times X)$ such that

(1) $p_{*}^{i} v_{n}=\eta$ for all $n$ and $i=1,2$.

(2) $\int_{X \times X} f(x) g(y) d v_{n}(x, y) \rightarrow \int_{X} f g d \eta$ for all $f, g \in B(X)$.

(3) $\left\|(\theta \times \theta)_{*} v_{n}-v_{n}\right\| \rightarrow 0$ for every $\theta \in[R]$.

(4) $v_{n}\left(\Delta_{X}\right) \not \rightarrow 1$.

After passing to a subsequence we can assume that $c_{n}=1-v_{n}\left(\Delta_{X}\right)$ satisfy $c=\inf c_{n}>0$. Define $\mu_{n} \in \mathcal{M}(X \times X)$ by $\mu_{n}(A)=c_{n}^{-1} v_{n}\left(A \backslash \Delta_{X}\right)$ for every Borel set $A \subset X \times X$. We have that

$\left(1^{\prime}\right) p_{*}^{i} \mu_{n} \leqslant c^{-1} \eta$ for all $n$ and $i=1,2$.

(2') $\mu_{n}(A \times(X \backslash A)) \rightarrow 0$ for any Borel set $A \subset X$.

(3') $\left\|(\theta \times \theta)_{*} \mu_{n}-\mu_{n}\right\| \rightarrow 0$ for every $\theta \in[R]$.

(4') $\mu_{n}\left(\Delta_{X}\right)=0$ for all $n$.

Let $\theta \in[R]$. After modifying $\theta$ on a null set, we can assume that $\theta(x) \in \Gamma x \Lambda$ for all $x \in X$. This allows us to define $w_{\theta}=\left(w_{\theta}^{1}, w_{\theta}^{2}\right): X \rightarrow \Gamma \times \Lambda$ through the formula $\theta(x)=w_{\theta}(x) \cdot x=w_{\theta}^{1}(x) x w_{\theta}^{2}(x)^{-1}$ for every $x \in X$. Using $w_{\theta}^{1}$ we construct a Borel isomorphism $\hat{\theta}$ of $X \times \mathbf{P}(\mathfrak{g})$ by letting $\hat{\theta}(x, Y)=\left(\theta(x), \operatorname{Ad}\left(w_{\theta}^{1}(x)\right)(Y)\right)$.

We split the rest of the proof into several steps.

Step 1. There exists $\zeta \in \mathcal{M}(X \times \mathbf{P}(\mathrm{g}))$ such that $\hat{\theta}_{*} \zeta=\zeta$ for all $\theta \in[R]$.

Proof of Step 1. For every $n$, let $\zeta_{n}=\pi_{*} \mu_{n}$. Let $\theta \in[R]$. To prove this step we first show that $\zeta_{n}$ are "almost $\hat{\theta}$-invariant" and then argue that any limit point $\zeta$ of $\left\{\zeta_{n}\right\}_{n \geqslant 1}$ satisfies the conclusion. We first claim that

$$
\left\|\hat{\theta}_{*} \zeta_{n}-\zeta_{n}\right\| \rightarrow 0
$$

By Lemma $\mathrm{F}$ (which applies as $\left(1^{\prime}\right),\left(2^{\prime}\right)$ and $\left(4^{\prime}\right)$ hold true), the set $D$ of $(x, y) \in$ $X \times X$ such that $\rho(\theta(x), \theta(y))=\operatorname{Ad}\left(w_{\theta}^{1}(x)\right)(\rho(x, y))$ satisfies $\mu_{n}(D) \rightarrow 1$. Since $(x, y) \in D$ if and only if $(\hat{\theta} \circ \pi)(x, y)=(\pi \circ(\theta \times \theta))(x, y)$, condition ( $\left.3^{\prime}\right)$ gives (c).

Now, since $\zeta_{n}$ are Borel probability measures on the locally compact metrizable space $X \times \mathbf{P}(\mathfrak{g})$, we can view them as elements of $C_{0}(X \times \mathbf{P}(\mathfrak{g}))^{*}$ (the dual of the algebra of continuous complex-valued functions on $X \times \mathbf{P}(\mathrm{g})$ which vanish at infinity). Since the unit ball of $C_{0}(X \times \mathbf{P}(\mathfrak{g}))^{*}$ is compact metrizable in the weak-* topology, by Riesz' representation theorem we can find a subsequence $\left\{\zeta_{n_{k}}\right\}_{k \geqslant 1}$ and a positive Borel measure $\zeta$ on $X \times \mathbf{P}(\mathrm{g})$ such that $\int_{X \times \mathbf{P}(\mathrm{g})} f d \zeta_{n_{k}} \rightarrow \int_{X \times \mathbf{P}(\mathrm{g})} f d \zeta$ for every $f \in C_{0}(X \times \mathbf{P}(\mathrm{g}))$.

Towards showing that $\zeta$ satisfies the conclusion, note first that $0 \leqslant \zeta(X \times \mathbf{P}(\mathrm{g})) \leqslant$ 1. Let $\varepsilon>0$. Since $X \times \mathbf{P}(\mathrm{g})$ is a metrizable space, by Lusin's theorem we can find a closed subset $X_{0} \subset X$ such that $\eta\left(X \backslash X_{0}\right) \leqslant \varepsilon$ and $\left.w_{\theta}\right|_{X_{0}}: X_{0} \rightarrow \Gamma \times \Lambda$ is 
continuous. Condition (1') implies that $\zeta_{n}\left(\left(X \backslash X_{0}\right) \times \mathbf{P}(\mathfrak{g})\right)=\mu_{n}\left(\left(X \backslash X_{0}\right) \times X\right) \leqslant$ $c^{-1} \eta\left(X \backslash X_{0}\right) \leqslant c^{-1} \varepsilon$ for every $n$. Thus $\zeta_{n}\left(X_{0} \times \mathbf{P}(\mathrm{g})\right) \geqslant 1-c^{-1} \varepsilon$ and since $X_{0} \times \mathbf{P}(\mathfrak{g})$ closed, we deduce that $\zeta\left(X_{0} \times \mathbf{P}(\mathfrak{g})\right) \geqslant 1-c^{-1} \varepsilon$. As $\varepsilon>0$ is arbitrary, this proves that $\zeta$ is a probability measure.

By (c), in order to prove that $\hat{\theta}_{*} \zeta=\zeta$ it suffices to show that $\int(f \circ \hat{\theta}) d \zeta_{n_{k}} \rightarrow$ $\int(f \circ \hat{\theta}) d \zeta$ for every $f \in C_{0}(X \times \mathbf{P}(\mathfrak{g}))$ with $\|f\|_{\infty} \leqslant 1$. Note that the restriction of $\hat{\theta}$ to $X_{0} \times \mathbf{P}(\mathfrak{g})$ continuous. Let $h \in C_{0}(X \times \mathbf{P}(\mathfrak{g}))$ such that $\|h\|_{\infty} \leqslant 1$ and $\left.h\right|_{X_{0} \times \mathbf{P}(\mathrm{g})}=$ $\left.(f \circ \hat{\theta})\right|_{X_{0} \times \mathbf{P}(\mathfrak{g})}$. Since $\zeta\left(\left(X \backslash X_{0}\right) \times \mathbf{P}(\mathfrak{g})\right) \leqslant c^{-1} \varepsilon$ and $\zeta_{n}\left(\left(X \backslash X_{0}\right) \times \mathbf{P}(\mathfrak{g})\right) \leqslant c^{-1} \varepsilon$, we have that

$$
\begin{aligned}
& \left|\int_{X \times \mathbf{P}(\mathrm{g})}(f \circ \hat{\theta}) d \zeta_{n_{k}}-\int_{X \times \mathbf{P}(\mathrm{g})}(f \circ \hat{\theta}) d \zeta\right| \\
& \quad \leqslant 4 c^{-1} \varepsilon+\left|\int_{X \times \mathbf{P}(\mathrm{g})} h d \zeta_{n_{k}}-\int_{X \times \mathbf{P}(\mathrm{g})} h d \zeta\right| .
\end{aligned}
$$

Since $\int_{X \times \mathbf{P}(\mathrm{g})} h d \zeta_{n_{k}} \rightarrow \int_{X \times \mathbf{P}(\mathrm{g})} h d \zeta$ and $\varepsilon>0$ is arbitrary, this concludes the proof of Step 1 .

Next, by disintegrating $\zeta$, we derive the following:

Step 2. There exist an $R$-invariant Borel set $X_{0} \subset X$ with $\eta\left(X_{0}\right)>0$ and a Borel function $\zeta: X_{0} \rightarrow \mathcal{M}(\mathbf{P}(\mathfrak{g}))$ such that for all $\theta \in[R]$ we have $\zeta(\theta(x))=$ $\operatorname{Ad}\left(w_{\theta}^{1}(x)\right)_{*} \zeta(x)$ for $\eta$-a.e. $x \in X_{0}$.

Proof of Step 2. Since $p_{*}^{1} \mu_{n} \leqslant c^{-1} \eta$ and $\pi(x, y)=(x, \rho(x, y))$, we deduce that the push forward of $\zeta_{n}$ onto the $X$-coordinate is $\leqslant c^{-1} \eta$. Thus, the push forward of $\zeta$ onto the $X$-coordinate, denoted $\tilde{\eta}$, satisfies $\tilde{\eta} \leqslant c^{-1} \eta$. By using Step 1, we obtain that $\tilde{\eta}$ is $R$-invariant. Disintegrate $\zeta=\int_{X} \zeta(x) d \tilde{\eta}(x)$, where $\zeta(x) \in \mathcal{M}(\mathbf{P}(\mathfrak{g}))$ for all $x \in X$ (see e.g. [KM04], Theorem 3.3). By using Step 1 and the fact that $\tilde{\eta}$ is $R$-invariant, the uniqueness of the disintegration implies that

$$
\zeta(\theta(x))=\operatorname{Ad}\left(w_{\theta}^{1}(x)\right)_{*} \zeta(x) \text { for } \tilde{\eta} \text {-a.e. } x \in X \text { and all } \theta \in[R] .
$$

Denote by $X_{0} \subset X$ the support of $\tilde{\eta}$ and notice that it is $R$-invariant. Since $\tilde{\eta} \leqslant c^{-1} \eta$ we obtain that $\eta\left(X_{0}\right)>0$ and the conclusion follows.

In the second half of the proof, we use Step 2 (and an analogous identity for $w_{\theta}^{2}$ ) to deduce that $\left.R\right|_{X_{0}}$ is hyperfinite. We first do this under the additional assumption that $\left.R\right|_{X_{0}}$ is ergodic with respect to $\eta$ and then treat the general case (see the end of the proof).

By using the fact that the action $H \curvearrowright \mathcal{M}(\mathbf{P}(\mathrm{g}))$ is smooth, we further obtain:

Step 3. There exist an amenable subgroup $P<H$ and a Borel function $\phi: X_{0} \rightarrow$ $H / P$ such that for all $\theta \in[R]$ we have $\phi(\theta(x))=w_{\theta}^{1}(x) \phi(x)$ for $\eta$-a.e. $x \in X_{0}$. 
Proof of Step 3. Since the adjoint action of $H$ on $g$ is linear, by [Zi84], Corollary 3.2.12, the action of $H$ on $\mathcal{M}(\mathbf{P}(\mathfrak{g}))$ is smooth (recall from [Zi84], Definition 2.1.9, that a Borel action $H \curvearrowright Z$ is smooth if there exists a sequence of Borel sets $W_{n} \subset Z / H$ which separate points). Since $\left.R\right|_{X_{0}}$ is ergodic, by Step 2 we deduce that $\zeta_{x}$ lies in a single $H$-orbit, on a co-null subset of $X_{0}$. In other words, there exists $\xi \in \mathcal{M}(\mathbf{P}(\mathfrak{g}))$ such that $\zeta_{x} \in H \xi$ for $\eta$-almost every $x \in X_{0}$. Identify $H \xi$ with $H / P$, where $P$ denotes the stabilizer of $\xi$ in $H$.

We claim that $P$ is amenable. By [Sh99], Theorem 3.11, $P$ has a normal cocompact subgroup $P_{0}$ which fixes every point in the support of $\xi$. Thus, if $Y \in \mathfrak{g} \backslash\{0\}$ is such that $p(Y) \in \mathbf{P}(\mathrm{g})$ is in the support of $\xi$, then there exists a homomorphism $\chi: P_{0} \rightarrow \mathbb{R}^{*}$ such that $\operatorname{Ad}(\gamma)(Y)=\chi(\gamma) Y$ for all $\gamma \in P_{0}$. Now, $P_{1}=\operatorname{ker}(\chi)$ stabilizes $Y$ and our assumption implies that $P_{1}$ is amenable. Since $\chi$ is continuous and $\mathbb{R}^{*}$ is amenable, we deduce that $P_{0}$ is amenable. Finally, as $P_{0}$ is co-compact in $P$, it follows that $P$ is amenable and the conclusion follows.

Now, define $r^{\prime}: G \times G \rightarrow G$ as $r^{\prime}(x, y)=x^{-1} y$. Let $\rho^{\prime}=p \circ q \circ r^{\prime}:(G \times G) \backslash$ $\Delta \rightarrow \mathbf{P}(\mathfrak{g})$ and $\pi^{\prime}:(G \times G) \backslash \Delta \rightarrow G \times \mathbf{P}(\mathfrak{g})$ be given by $\pi^{\prime}(x, y)=\left(x, \rho^{\prime}(x, y)\right)$. Repeating the above argument with $r^{\prime}, \rho^{\prime}, \pi^{\prime}$ instead of $r, \rho, \pi$ yields an amenable subgroup $Q<K$ and a Borel function $\psi: X_{0} \rightarrow K / Q$ and such that $\psi(\theta(x))=$ $w_{\theta}^{2}(x) \psi(x)$ for almost every $x \in X_{0}$, for all $\theta \in[R]$ (note that $\tilde{\eta}$ is the weak-* limit of $p_{1}^{*} \mu_{n_{k}}$, so its support, $X_{0}$, does not depend on the definition of $r$ ).

Set $Z=H / P \times K / Q$ and $\tau:=(\phi, \psi): X_{0} \rightarrow Z$. Then $\tau(\theta(x))=w_{\theta}(x) \tau(x)$ for almost every $x \in X_{0}$, for all $\theta \in[R]$. Here on $Z=(H \times K) /(P \times Q)$ we consider the left multiplication action of $\Gamma \times \Lambda$. Since $P \times Q$ is amenable and $\Gamma \times \Lambda$ is discrete, this action is topologically amenable, in the sense of [An02] (see the proof of [BO08], Theorem 5.4.1). This fact allows us to derive the following:

Step 4. $\left.R\right|_{X_{0}}$ is hyperfinite.

Proof of Step 4. This is a consequence of Proposition 3.6 in [Io10]. For the reader's convenience we provide a self-contained argument. Fix a sequence $\left\{\theta_{i}\right\}_{i \geqslant 1} \subset[R]$ such that $R=\bigcup_{i \geq 1}\left\{\left(\theta_{i}(x), x\right) \mid x \in X\right\}$. Define $w: R \rightarrow \Gamma \times \Lambda$ by $w(x, y)=$ $w_{\theta_{i}}(y)$, where $i$ is the least integer with $x=\theta_{i}(y)$. Then $\tau: X_{0} \rightarrow Z$ satisfies

$$
\tau(x)=w(x, y) \tau(y) \quad \text { for } \eta \text {-almost every }\left.(x, y) \in R\right|_{X_{0}} .
$$

Since the action $\Gamma \times \Lambda \curvearrowright Z$ is topologically amenable, by Connes-FeldmanWeiss' theorem ([CFW81]), its orbit equivalence relation $T$ is hyperfinite with respect to any measure on $Z$. Let $\left.\eta\right|_{X_{0}}$ be the measure on $X_{0}$ given by $\left.\eta\right|_{X_{0}}(A)=\eta\left(A \cap X_{0}\right)$. Then we can find an increasing sequence $T_{n}$ of finite equivalence relations on $Z$ such that $T=\bigcup_{n \geqslant 1} T_{n}$, up to $\tau_{*}\left(\left.\eta\right|_{X_{0}}\right)$-null sets.

For every $n \geqslant 1$, set $R_{n}=\left\{\left.(x, y) \in R\right|_{X_{0}} \mid(\tau(x), \tau(y)) \in T_{n}\right\}$. Then $R_{n}$ is an increasing sequence of subequivalence relations of $\left.R\right|_{X_{0}}$. By (d) we have that $\bigcup_{n \geqslant 1} R_{n}=\left.R\right|_{X_{0}}$, up to $\eta$-null sets. Thus, to show that $\left.R\right|_{X_{0}}$ is hyperfinite, it is 
enough to argue that $R_{n}$ is hyperfinite for all $n \geqslant 1$. Now, if $R_{0}=\left\{\left.(x, y) \in R\right|_{X_{0}} \mid\right.$ $\tau(x)=\tau(y)\}$, then $R_{0}$ has finite index in each $R_{n}$. Therefore, we can further reduce to proving that $R_{0}$ is hyperfinite.

Let us first prove this under the additional assumption that $R_{0}$ is ergodic. Then we can find $\tau \in Z$ such that $\tau(x)=\tau$ for almost every $x \in X_{0}$. Denote by $L$ the stabilizer of $\tau$ in $\Gamma \times \Lambda$ and by $S_{0}$ the orbit equivalence relation of the action $L \curvearrowright G$ (recall that $\Gamma \times \Lambda$ acts on $G$ by left-right multiplication). By using (d) we derive that $w(x, y) \in L$ for almost every $(x, y) \in R_{0}$. Thus, $\left.R_{0} \subset S_{0}\right|_{X_{0}}$. On the other hand, since $P$ an $Q$ are amenable, it follows that $L$ is amenable. Thus, $S_{0}$ and $R_{0}$ are hyperfinite ([CFW81]).

If $R_{0}$ is not necessarily ergodic, we consider its ergodic decomposition. Let $\mathcal{M}$ be the set of ergodic $R_{0}$-invariant probability measures on $X_{0}$ (viewed as a Borel subset as $\mathcal{M}\left(X_{0}\right)$ ). Then there is an $R_{0}$-invariant Borel map $m: X_{0} \rightarrow \mathcal{M}$ such that $\left.\eta\right|_{X_{0}}=\left.\int_{X_{0}} m(z) d \eta\right|_{X_{0}}(z)$. Equation (d) implies that the set of $z \in X_{0}$ such that $\tau(x)=w(x, y) \tau(y)$, for $m(z)$-almost every $(x, y) \in R_{0}$, has full measure. Since $m(z)$ is $R_{0}$-ergodic, arguing as in the previous paragraph yields that $R_{0}$ is hyperfinite with respect to $m(z)$ for $\eta$-almost every $z \in X_{0}$. Since $\left.\eta\right|_{X_{0}}=\left.\int_{X_{0}} m(z) d \eta\right|_{X_{0}}(z)$, we conclude that $R_{0}$ is hyperfinite.

Finally, if $\left.R\right|_{X_{0}}$ is not ergodic, then one proceeds as in the last paragraph. Consider the ergodic decomposition $\left.\eta\right|_{X_{0}}=\left.\int_{X_{0}} m(z) d \eta\right|_{X_{0}}(z)$, where $m(z)$ are ergodic $\left.R\right|_{X_{0}}{ }^{-}$ invariant probability measures. Then the identity from Step 2 (and the analogous identity for $w_{\theta}^{2}$ obtained by replacing $\rho$ with $\rho^{\prime}$ ) holds when $\eta$ is replaced with $m(z)$ for almost every $z \in X_{0}$. Now, for such $z$, the above proof yields that $\left.R\right|_{X_{0}}$ is $m(z)$-hyperfinite. Finally, this gives that $\left.R\right|_{X_{0}}$ is $\left.\eta\right|_{X_{0}}$-hyperfinite.

We are now ready to prove the results announced in the introduction.

Proof of Theorem A. The first part is immediate by Theorem D. For the moreover part, recall Kazhdan's result: any connected semisimple Lie group $G$ with finite center whose simple factors have real-rank $\geqslant 2$ has property ( $T$ ) ([Ka67], see [Zi84], Theorem 7.4.2).

Proof of Corollary B. Denote by $\mu$ the probability measure on $X$. Let $(Y, v)$ be a pmp $\Gamma$-space and $p: X \times Y \rightarrow G / \Lambda$ be a measurable, quotient $\Gamma$-map. If $A:=$ $\left\{\left(x_{1}, x_{2}, y\right) \in X \times X \times Y \mid p\left(x_{1}, y\right)=p\left(x_{2}, y\right)\right\}$, then the conclusion is equivalent to $(\mu \times \mu \times v)(A)=1$. This implies that we may assume that the action $\Gamma \curvearrowright(Y, v)$ is ergodic.

Now, the action $\Gamma \curvearrowright\left(G / \Lambda, m_{G / \Lambda}\right)$ is rigid by Theorem A. By [Io09], Proposition 3.3, we obtain that $A$ has positive measure. Since $\Gamma \cdot i$ is infinite for all $i \in I$, the action $\Gamma \curvearrowright(X, \mu)$ is weakly mixing. Hence, the product action of $\Gamma$ on $X \times X \times Y$ is ergodic. Since $A$ is invariant under this action, the conclusion follows. 
Remark. In the case $Y$ is a single point space, Corollary B has been first proved by Furman in [Fu07], Remark 1.15 (2), by using entropy. When $\Gamma$ has property (T), this also follows from [Fu07], Theorem 1.14, by using Popa's cocycle superrigidity theorem.

Proof of Theorem C. Let $X \subset G$ be a fundamental set for the right $\Lambda$-action endowed with the probability measure $\left.m(X)^{-1} m\right|_{X}$. Set $T=\{(x, y) \in X \times X \mid x \in \Gamma y \Lambda\}$. Since the stabilizers under the adjoint action of $G$ of non-zero points in its Lie algebra $g$ are amenable, Theorem $E$ implies that any subequivalence relation $R \subset T$, admits an $R$-invariant measurable partition $X=X_{0} \cup X_{1}$ such that $\left.R\right|_{X_{0}}$ is hyperfinite and $\left.R\right|_{X_{1}}$ is rigid. Since $\phi: G / \Lambda \ni x \Lambda \rightarrow x \Lambda \cap X \in X$ is a measure preserving isomorphism with $\phi(S)=T$, we are done.

\section{References}

[An02] C. Anantharaman-Delaroche, Amenability and exactness for dynamical systems and their $C^{*}$-algebras. Trans. Amer. Math. Soc. 354 (2002), 4153-4178. Zbl 1035.46039 MR 1926869

[BO08] N. P. Brown and N. Ozawa, $C^{*}$-algebras and finite-dimensional approximations. Grad. Stud. Math. 88, Amer. Math. Soc., Providence, RI, 2008. Zbl 1160.46001 MR 2391387

[CFW81] A. Connes, J. Feldman, and B. Weiss, An amenable equivalence relation is generated by a single transformation. Ergodic Theory Dynamical Systems 1 (1981), 431-450. Zbl 0491.28018 MR 662736

[CJ85] A. Connes and V. Jones, Property $T$ for von Neumann algebras. Bull. London Math. Soc. 17 (1985), 57-62. Zbl 1190.46047 MR 766450

[Co99] J. B. Conway, A course in operator theory. Grad. Stud. Math. 21, Amer. Math. Soc., Providence, RI, 2000. Zbl 0936.47001 MR 1721402

[FM77] J. Feldman and C. C. Moore, Ergodic equivalence relations, cohomology, and von Neumann algebras. II. Trans. Amer. Math. Soc. 234 (1977), 325-359. Zbl 0369.22010 MR 0578730

[Fi10] P. Fima, Kazhdan's property $T$ for discrete quantum groups. Internat. J. Math. 21 (2010), 47-65. Zbl 1195.46072 MR 2642986

[Fu07] A. Furman, On Popa's cocycle superrigidity theorem. Internat. Math. Res. Notices 2007 (2007), no. 19, Art. ID rnm073. Zbl 1134.46043 MR 2359545

[Ga11] D. Gaboriau, Free product actions with relative property (T) and trivial outer automorphism groups. J. Funct. Anal. 260 (2011), 414-427. Zbl 1211.46071 MR 2737406

[GP05] D. Gaboriau and S. Popa, An uncountable family of nonorbit equivalent actions of $\mathbb{F}_{n}$. J. Amer. Math. Soc. 18 (2005), 547-559. Zbl 1155.37302 MR 2138136

[Io09] A. Ioana, Non-orbit equivalent actions of $\mathbb{F}_{n}$. Ann. Sci. École Norm. Sup. (4) 42 (2009), 675-696. Zbl 1185.37009 MR 2568879 
[Io10] A. Ioana, Relative property (T) for the subequivalence relations induced by the action of $\mathrm{SL}_{2}$ (Z) on $\mathbb{T}^{2}$. Adv. Math. 224 (2010), 1589-1617.Zbl 1202.46082 MR 2646305

[Io11] A. Ioana, Orbit inequivalent actions for groups containing a copy of $\mathbb{F}_{2}$. Invent. Math. 185 (2011), 55-73. Zbl 1230.37010 MR 2810796

[Ka67] D. A. Kazhdan, Connection of the dual space of a group with the structure of its close subgroups. Funkcional. Anal. i Priložen. 1 (1967), 71-74; English transl. Funct. Anal. Appl. 1 (1967), 63-65. Zbl 0168.27602 MR 0209390

[KM04] A. S. Kechris and B. D. Miller, Topics in orbit equivalence. Lecture Notes in Math. 1852, Springer-Verlag, Berlin 2004. Zbl 1058.37003 MR 2095154

[Ma82] G. A. Margulis, Finitely-additive invariant measures on Euclidean spaces. Ergodic Theory Dynam. Systems 2 (1982), 383-396 (1983). Zbl 0532.28012 MR 721730

[MvN36] F. J. Murray and J. Von Neumann, On rings of operators. Ann. of Math. (2) 37 (1936), 116-229. JFM 62.0449.03

[Po06] S. Popa, On a class of type $\mathrm{II}_{1}$ factors with Betti numbers invariants. Ann. of Math. (2) 163 (2006), 809-899. Zbl 1120.46045 MR 2215135

[PV10] S. Popa and S. Vaes, Actions of $\mathbb{F}_{\infty}$ whose $\mathrm{II}_{1}$ factors and orbit equivalence relations have prescribed fundamental group. J. Amer. Math. Soc. 23 (2010), 383-403. Zbl 1202.46069 MR 2601038

[Sh99] Y. Shalom, Invariant measures for algebraic actions, Zariski dense subgroups and Kazhdan's property (T). Trans. Amer. Math. Soc. 351 (1999), 3387-3412. Zbl 0932.22007 MR 1615966

[Zi84] R. J.Zimmer, Ergodic theory and semisimple groups. Monogr. Math. 81, Birkhäuser, Basel 1984. Zbl 0571.58015 MR 776417

Received November 4, 2010; revised July 27, 2011

A. Ioana, Department of Mathematics, UCSD, La Jolla, CA 92093-0112, U.S.A.

E-mail: aioana@math.ucsd.edu

Y. Shalom, UCLA Mathematics Department, Los Angeles, CA 90095-1555, U.S.A.

E-mail: shalom@math.ucla.edu 